\title{
Implementing the role of patient-navigator nurse at a university hospital centre
}

By Lise Fillion, Marie de Serres, Richard Lapointe-Goupil, Isabelle Bairati, Pierre Gagnon, Michèle Deschamps, Josée Savard, François Meyer, Luc Bélanger and Georges Demers

\section{Acknowledgement}

We wish to thank Nadine Bernier, Jean-David Gaudreau, Annick Millette, and Marie-Anik Robitaille for helping to coordinate this project and participating in the qualitative analysis of data in order to obtain interrater agreement; Louise Saint-Laurent for proofing the article and for her expertise in qualitative approach and health care organization; all stakeholders and administrators in the hospital

Lise Fillion, RN, PhD, Faculté des Sciences Infirmières [Faculty of Nursing], Université Laval, Quebec City, QC, Centre de recherche en cancérologie [Cancer Research Centre], Université Laval, Quebec City, QC, Équipe de recherche de la Maison Michel-Sarrazin [Michel-Sarrazin Place Research Team], Quebec, $Q C$.

Marie de Serres, RN, MScN, Centre hospitalier universitaire de Québec [Quebec City University Hospital Centre], Pavillon Hôtel-Dieu de Québec, Quebec.

Richard Lapointe-Goupil, MPs, PhD(c), Centre de recherche en cancérologie [Cancer Research Centre], Université Laval, Quebec City, QC.

Isabelle Bairati, $M D, P h D$, Centre de recherche en cancérologie [Cancer Research Centre], Université Laval, Quebec City, QC

Pierre Gagnon, MD, FRCPC, Centre de recherche en cancérologie [Cancer Research Centre], Université Laval, Quebec City, QC, Équipe de recherche de la Maison Michel-Sarrazin [MichelSarrazin Place Research Team], Québec, QC, Centre hospitalier universitaire de Québec [Quebec City University Hospital Centre], Pavillon Hôtel-Dieu de Québec, Quebec.

Michèle Deschamps, $R N$, PhD, Direction de santé publique de Montréal [Montreal Public Health Division], QC.

Josée Savard, PhD, Centre de recherche en cancérologie [Cancer Research Centre], Université Laval, Quebec City, QC.

François Meyer, MD, PhD, Centre de recherche en cancérologie [Cancer Research Centre], Université Laval, Quebec City, QC.

Luc Bélanger, $M D, P h D$, Centre de recherche en cancérologie [Cancer Research Centre], Université Laval, Quebec City, QC.

Georges Demers, MD, FRCPC, Centre hospitalier universitaire de Québec [Quebec City University Hospital Centre], Pavillon Hôtel-Dieu de Québec, Quebec.

All correspondence should be addressed to Dr. Lise Fillion, E-mail: lise.fillion@fsi.ulaval.ca centre who believed and participated in the approach; the reviewers of the article. This study was funded by RRSSS-03 (Regional Health and Social Services Board \#03) and the CCQLC (Quebec Coordination Centre for Cancer Control). The study was also facilitated through the collaboration and ad hoc funding of the DSI (Nursing Branch) of CHUQ-HDQ. It was also supported by a research grant to the first author.

Key words: oncology, patient navigator, cancer control program, implementation, psychosocial adjustment, program evaluation

\section{Abstract}

A profile of the role and functions of an oncology patient-navigator nurse $(O P N)$ and the preliminary phases to implementing this role within a team specializing in oncology are first presented. This is followed by a qualitative study that provides a descriptive assessment for implementing an initial OPN in the head and neck oncology area of a university hospital centre (UHC) with a supraregional model for oncology. Three groups of stakeholders (individuals with cancer and families, caregivers, network partners) were interviewed on three occasions: before, during and after implementation. The results show that this new role can be integrated within a team specializing in oncology. The beneficial effects of this role on the process of adaptation to illness, interdisciplinary work and continuity of care are described. Several recommendations are formulated, one being the importance of situating the implementation process from an organizational change perspective.

In Canada, it is expected that the number of new cancer cases will increase by $60 \%$ in the next two decades. Cancers of the head and neck area represent $4.1 \%$ of all cancers and are 2.4 times more frequent among men than they are among women (National Cancer Institute of Canada, 2005). This type of cancer includes oral cancers and cancers of the pharynx and larynx.

In general, individuals with cancer and their families feel powerless, without resources, abandoned to their own devices, and don't know who to turn to when they have questions. These feelings are particularly vivid during critical periods such as communication of diagnosis (Fraser, 1995). These individuals need to be accompanied and supported throughout the long therapeutic journey and to be provided with adapted information regarding the disease, treatments and available resources. They also wish for better communication with specialists (Comité consultatif sur le cancer, 1997). Individuals with cancer frequently deplore the fact that they must be seen by a large number of caregivers at several facilities and the lack of coordination between the phases of the care trajectory (Turgeon, Dumont, St-Pierre, Sévigny, \& Vézina, 2004). This is particularly true for patients with a head and neck cancer who must cope with the additional treatment sequelae such as a changed appearance, loss of speech and decreased quality of interaction (Kugaya, Akechi, Okamura, Mikami, \& Uchitomi, 1999). 


\section{Role and functions of the oncology patient-navigator nurse (OPN)}

Several roles connected to that of patient navigator $(\mathrm{PN})$ have been described in the literature and are known under the terms: case manager, clinical coordinator, cancer support nurse, followup nurse, advanced practice nurse, breast specialist, breast nurse, breast cancer coordinator, patient navigator (Farber, Deschamps, \& Cameron, 2002). Despite differences in their descriptions, all these roles fit in with the concept of a holistic approach to the person centring on quality of life of the person with cancer (Fillion, Morin \& Saint-Laurent, 2000). The role of a PN is related to the role of a third generation case manager as described by Anderson-Loftin (1999), which values empowering the patient and humanizing the care. Beyond efficiently coordinating the treatment trajectory, the PN promotes continuity of care by considering both the needs of the patient with cancer and the system's characteristics. In some way, the PN becomes an advocate for the patient and the multidisciplinary team. The role begins as soon as the diagnosis has been announced and finishes at the end of the care trajectory (recovery, reintegration into environments, palliative care).

The title of oncology patient-navigator nurse (OPN) has recently been selected to identify this role (de Serres \& Rochette, 2004). The role of OPN is often carried in the community, for example, Interlink in Ontario (Howell \& Jackson, 1998). The nurse is qualified to assess psychosocial and physical needs (Bottomley \& Jones, 1997; Conger \& Craig, 1998; Corner, 1999; Goldberg, 1994; Nickel et al., 1996; Nolan, Harris, Kufta, Opfer, \& Turner, 1998) and advocate on the patient's behalf (Agretelis, 1997; Ambler et al., 1999; Weekes, 1997). In Canada, oncology certification prepares nurses particularly well for these more specialized functions (Fitch \& Mings, 2003).

To take on the role of an OPN (de Serres \& Beauchesne, 2000; Fillion et al., 2000), the candidates must demonstrate: 1) competencies in oncology, 2) a training which enables them to assess the biological, psychosocial and spiritual aspects of individuals with cancer and their families, 3) a knowledge of health promotion and family therapy, 4) skills in communication, helping relationships, teamwork and problem-solving, 5) professional leadership (being familiar with and mobilizing resources).

Four functions are linked to the role of OPN for individuals with cancer and their families: assess the needs and follow up on the interventions implemented through the initial assessment; inform and teach; provide special support and attention and coordinate and promote continuity of care, services, information and therapeutic relationship (de Serres \& Beauchesne, 2000). Both the functions and scope of the role must remain flexible in order to respect the culture and service model in the setting and avoid duplication of services and roles among the various caregivers.

Considering the relevancy of this new role to oncology and the lack of information on its implementation in existing teams, it seems necessary to document the first implementation initiatives with a team specializing in oncology. Inspired by the evaluation approach established by Haddock, Johnson, Cavanaugh and Stewart (1997) for an oncology case management program, we decided to propose and apply a role and function profile for an OPN in one area of oncology, and to produce a descriptive and qualitative assessment of the implementation process.

\section{Conceptual frameworks}

Two conceptual frameworks were selected to explore these issues. The first one which deals with organizational changes (Robert, 2000) underscores the importance of properly paving the way before the implementation itself by explaining the vision behind the new role, identifying the changes required to move from the current to the desired situation and providing a strategic justification for this role based on criteria for legitimacy, relevancy, credibility, pragmatism and capacity for change of the organization. This framework guided the establishment of a preparation phase for the organizational change. The second framework (Patton, 1997; adapted by Fillion \& Morin, 2003) is also based on the theoretical concept of change and constitutes more of an operational framework for program assessment. It suggests studying the implementation process according to the seven dimensions described in Table One.

\section{Purpose and objectives}

This project aims to propose a profile for the role and function of an OPN and to assess its implementation in a specialized team within a university hospital centre (UHC) with a supraregional model for oncology, using a qualitative approach. The general objective for the assessment process is to provide a qualitative description of the implementation process of the OPN role and its effects. Specific objectives are to describe the following items according to the perceptions of the various stakeholders: 1) role and functions of an OPN, 2) role outcomes on the process of adaptation to illness for individuals with cancer and their families, and 3 ) role outcomes on continuity of care and services delivered by the UHC oncology interdisciplinary team and care network external partners.

\section{Method}

This section first presents the preliminary preparation phase to the implementation and then the selected method for the evaluation approach for the implementation process.

\section{Preparation phase to the implementation}

The project started with putting forward a profile for the role and functions of an OPN taken from the Quebec Cancer Control Program (PQLC) (as summed up in the introduction to this article) and establishing an implementation committee composed of representatives from the clinical, administrative and research sectors of the UHC. Subsequently, a series of information and consultation meetings took place with the various stakeholder groups in oncology (oncologists, nurses, technicians, Council of Nurses) to present the profile and vision for the OPN role proposed in the PQLC and to properly delineate the role within the institution. Participants were invited to provide feedback regarding the various aspects of the role and implementation process and determine in which area the OPN role should be first implemented. The head and neck area was chosen, and an area committee (representing the interdisciplinary team) was established to facilitate the operational definition of the role throughout the implementation process. A competition was started to select an individual with a bachelor of science in nursing, clinical experience in oncology and Canadian certification in oncology. A training program was then developed. This program's content and format involved the acquisition of the theoretical (courses and discussions) and practical (brief internships) knowledge related to the complete care trajectory for the head and neck area.

This preliminary phase allowed us to develop a vision, adjust the OPN role and functions in accordance with the UHC's resources and oncology multidisciplinary team, and put in place the structure for its implementation. The evaluation phase followed.

\section{Evaluation approach for the implementation process}

The evaluation approach required a descriptive, qualitative, single-case design with the selection of a single UHC. In order to increase the validity of the study, a triangulation of the stakeholders 
potentially affected by the change was completed (Poupart, Deslauriers, Groulx, Laperrière, Mayer, \& Pires, 1997). The sample was composed of three groups of participants: 1) individuals with head and neck cancers and their families who were seen by the OPN, 2) caregivers at the UHC, and 3) network partners. Data collection was completed on three occasions, i.e. before (T0), during (T1) and approximately one year after implementation (T2). The longitudinal nature of the data allows us to better describe the dynamic aspect of the implementation process. A research assistant collected the data using semi-structured interviews. These interviews were conducted with participants from each of the groups. The interview schedule contains a socio-demographic section and a section with semi-open questions developed from Patton's framework (1997). For example, in order to assess activities (dimension 2, see Table One), individuals with cancer are asked: "What has the OPN done for you? And for your family?"

Table One. Dimensions of Patton's Framework (1997), adapted by Fillion and Morin, 2003

Dimensions and Definitions $\quad$ Sample quotes

1. Origins: Understanding the

project and link to PQLC

2. Activities: What the OPN does (according to the 4 major functions proposed by the PQLC and narrowed down by de Serres and Beauchesne, 2000).

T1-PT16: I felt totally shattered. The words she was saying were such that I slowly calmed down until I could speak, because I was crying all the time.

T1-FAM07: It is the warmth, the confidence she brings us.

T2-RN-I83: The OPN plays a coordination role. She becomes the person referrals are made to. She is a first responder. She receives messages and calls, makes interviews and directs patients to the right resources. She is present at diagnosis. That's when she begins the initial information collections that yield a better knowledge of the patient. From the patient perspective, she helps orientation to resources. She also does some teaching about the surgery and treatments. She also has a support role.

T2-MDDMD-I82: She makes the link between the scientific data regarding the illness, medical work-up and treatment plans. She ensures that both the patient and their loved ones understand the interventions. She accompanies the patient and all the other caregivers throughout the trajectory, from the initial referral phase for an opinion to consultations, diagnostic and therapeutic process and follow-up. She fields the questions patients have along the way, even when they are outside the UHC.

X13-RN: The information is properly relayed because the client knows the OPN well and that she is the resource person. In some UHCs, one can never talk to the right person. When a patient has an OPN, I don't worry, as I know there is someone who is going to support me.

3. Participation: Stakeholders participation characteristics.

4. Reactions: Participants' attitudes toward the OPN.

T2-OP-I49: I get involved because of the nature of our work in rehabilitation where we see the importance of long-term care quality. It is useful and beneficial to have someone ensuring long-term follow-up.

T0-TEC-G2: It is useful, but she must be available otherwise it is an inconvenience. It is obvious that she should not be imposed on either.

T1-RN-G1: There were huge gaps in ORL. This is heaven-sent! We have a better knowledge of the issues through her data collection. It also reduced stress in patients. If our teaching is too fast for the patients, they know that the OPN will complete the information. It lessens the weight on our shoulders.

5. Changes in attitudes, knowledge and values.

T2-MDDMD-I50: I have a better understanding of some of the problems experienced by patients that they don't mention to us.

6. Behavioural and practical changes.

T2-MDDMD-I46: It allows each specialist to focus a bit more on their specialized tasks. It has helped the ORL services complex, a weighty organization with many caregivers, to function better.

T2-RN-I83: The OPN brings some recognition to what I currently do.

T2-OP-I34: For the team, I think it is the unifying role of the OPN that has the greatest potential and that role is not fully exploited right now.

T2-TEC-G1: I feel like we generally get more consideration from the nurses. I find we can talk as equals.

7. Impacts: Anticipated medium-term effects
T2-PT17: If I experience difficulties or problems, I am not going to mind; I am going to ask for her as she told me to. I have her business card. Even from here (from my distant region), I can communicate with her.

T2-OP-I49: Clinicians don't have enough time to perform support and follow-up services in the continuous and regular manner the OPN does.

T2-MDDMD-I87: She cares enough to ask if there is a family physician, making sure he is kept informed. She can help patients who live in outlying areas.

Note. OPN: oncology patient nurse; PQLC: French acronym of the Quebec Cancer Control Program. T0 = Time 0, T1 = Time 1, T2 = Time 2; PT = Patient; FAM = Family, RN = Nurse; OP = Other Professionals; TEC: Radiation oncology technologists; MDDMD = Physician or dentist; $\mathrm{I}=$ Individual interview; $\mathrm{G}=$ Group interview; $\mathrm{X}$ = before a number, refers to an external caregiver 
The group of individuals with cancer and their families was formed a few months after the OPN role had been implemented at a time determined by the research team. Accrual was done by the OPN following the initial assessment of the patients. Each of the recruited individuals was contacted by a research assistant to arrange a meeting for an interview lasting approximately 40 minutes, in a room reserved to that effect at the UHC. In a few cases, the interview was completed over the phone. For this first group, accrual ended with data saturation, i.e. when no new information can be obtained for any of the dimensions. The UHC stakeholder group was established before implementing the OPN role from a list drafted by the institution's implementation committee. All caregivers working in head and neck oncology were invited to participate in the study by the research assistant. The interview, which lasted approximately 50 minutes, took place in a dedicated room at the UHC or in the caregivers' offices. Caregivers were divided into the following categories: 1) nurses, 2) physicians and dentists, 3) radiation oncology technologists, and 4) other health professionals (nutritionists, speech therapists, social workers). The network partners group was created from the list of outside caregivers who were contacted at least once by the OPN during the course of the study. It was composed of nurses, physicians and other professionals (occupational therapists and nutritionists) working in the community (i.e. in the local community health centres) and collaborating with the UHC. The research assistant contacted the network partners to invite them to participate in the research project and to arrange a telephone interview lasting approximately 25 minutes. Generally, each group included the same members who were interviewed at the three measurement times. A few left when their functions no longer involved head and neck oncology while a few new ones joined in. This turnover affected mostly the nursing group. All participants were informed about the goals of the study and signed a consent form. The research project was approved by the UHC's research ethics board.

The content of the interviews was recorded, summarily transcribed and coded according to each of the seven dimensions in the conceptual framework for each group and sub-group. The coding process involved two coders until a consensus was reached regarding both the determination and classification according to the codes. In order to compare and integrate the various groups of stakeholders and the different measurement times, the comments made during the interviews were analysed in accordance with the approach suggested by Strauss and Corbin (1990), i.e. intra-group analysis, inter-group comparison and change over time. The results are presented for each of Patton's seven dimensions and interpreted in order to answer the three following research questions: 1) What is the perception of the OPN's role and functions and how did this perception emerge and evolve during implementation? (Dimensions 1 to 4 ); 2) How does the implementation of this new role contribute to the process of adaptation to illness for individuals with cancer and their families? (Dimensions 5, 6 and 7); 3) What is the perception of key stakeholders regarding the changes and effects of implementing the role on the continuity of care and services? (Dimensions 5, 6 and 7).

\section{Results}

The sample description is followed by the presentation of results according to the seven dimensions of the conceptual framework (see Table One for a list of quotes).

\section{Sample description}

The group of individuals with cancer $(n=19)$ was composed of a majority of men (74\%), with an average age of 60 and education at the secondary school level or less $(58 \%)$. Family members $(n=15)$ were mainly women (73\%) with an average age of 56 and education slightly above the secondary school level (53\%). The UHC stakeholders group $(n=47)$ was mainly composed of women $(76 \%)$ whose average age is 46 , two-thirds of which had a college or university education $(72 \%)$. Nurses made up a third of the sample $(33 \%)$, followed by radiation oncology technologists $(25 \%)$, physicians and dentists (21\%) and "other professionals" (21\%: nutritionists, social workers, speech therapists). The network partners group $(n=21)$ was generally composed of women $(81 \%)$, with an average age of 41 and holding a bachelor's degree $(67 \%)$. The network partners were mostly nurses $(71 \%)$, but also included physicians (10\%) and other professionals (29\%: nutritionists, occupational therapists).

\section{Perception of the origins of the implementation}

As the first dimension referred to the understanding of the OPN role and how it should be implemented in an evaluative research context, this dimension was only assessed among the UHC's caregivers. At both T0 and T1, the nurses' group and the physicians' and dentists' group reported they were well-informed regarding both the steps of the research project and the OPN's future functions. Regarding the technologists, their knowledge was limited at the three measurement points. The other professionals declared being moderately informed. One year after implementation (T2), comprehension of the OPN's role was becoming more precise for most participants, and the words they used to describe it presented a greater homogeneity.

\section{Perception regarding the activities and functions performed by the OPN}

The second dimension helped to describe the OPN's activities and functions. Comments were gathered amongst participants from all of the groups.

Individuals with cancer and their families. The majority of individuals with cancer and their families first described the support they received from the OPN. They especially appreciated the time they were granted, the information they were given and this caregiver's skills in communication and in a helping relationship. A large number perceived the OPN as someone who knows how to really listen, ask questions, and encourages individuals to verbalize concerns. They mentioned that a part of the support was linked to the teaching that was given in a timely fashion and the information presented in such a way that it could be understood. Individuals with cancer mentioned the help they had with making appointments and communicating with physicians.

UHC caregivers. Before implementation (T0), all caregivers thought that implementing the OPN's role would improve care coordination which, in turn, would facilitate standardizing the care trajectory. At Time 2, coordination was perceived as more centred on the needs of individuals with cancer (supportive care and attention, orientation, continuity of follow-up) than those of the system (standardization of the trajectory). The functions related to assessment, teaching and psychological support were also described.

Network partners. For nurses in local community health centres, the OPN was generally perceived as a resource person for individuals with cancer and for themselves as she has a clinical expertise which she shared with them. The coordination function of the role was also highlighted.

\section{Participation of the stakeholders with the OPN}

The third dimension dealt with the participation of the stakeholders in using the services or integrating the OPN within the healthcare teams. 
Individuals with cancer and their families. Individuals with cancer and their families turned to the OPN based on the nature and scope of their needs. Some patients never availed themselves of the OPN's services as their needs were met through other means.

UHC caregivers. Before the start of the project, all stakeholders wanted to get involved in implementing the OPN role to improve the quality of care. Both the motivation and interest were still present one year after implementation. Some caregivers declared they were engaging actively in implementing this new role as it helped reinforce the supraregional nature of oncology at their institution and improved the head and neck service hub.

Network partners. In general, caregivers at local community health centres participated well. Nurses and other professionals communicated with the OPN via phone, fax or e-mail while physicians used mainly the phone. However, it seemed that the lack of information on the OPN's functions limited these caregivers' participation in the integration of the role at the community level. Moreover, the participation of primary care physicians was limited. While they had been informed of the OPN's involvement in their patients' follow-up, very few of these physicians contacted her over the duration of the study.

\section{Stakeholders' reactions}

The fourth component was the participants' reaction to the implementation of an OPN.

Individuals with cancer and their families. Satisfaction was very high among individuals with cancer and their families. According to them, this professional contributes to humanizing heath care.

UHC caregivers. Even before implementation, UHC caregivers demonstrated a favourable attitude towards this new role. However, some feared that the availability of the OPN would rapidly become a problem due to work overload, and that she would become one more caregiver in an already complex care organization. But this fear disappeared after the role was implemented. Caregivers believed that everyone - caregivers, individuals with cancer and their families - benefited from the OPN presence. However, a few nurses indicated concerns over the dependency relationship with the OPN and the lack of accountability the individual with cancer could develop for the OPN regarding their care. One year after implementation, the reaction to the OPN's role was essentially positive. This positive reaction also existed among the network partners.

\section{Perception of changes in attitude and behaviours}

This section dealt with the perception of the changes associated with the presence of an OPN and was recorded at the level of participants' values, attitudes and cognitions and at the level of shortand medium-term behavioural changes. These types of changes correspond to the last three dimensions of Patton's framework (see Table One).

Individuals with cancer and their families. Individuals with cancer and their families spontaneously reported that the OPN helped them adjust to the illness, i.e. coping with a cancer diagnosis, gaining a better understanding of the illness and better preparing for treatments. For some, the OPN's presence helped them mobilize their coping strategies. The OPN fostered the use of problem-solving, helped others find ways of entertaining themselves and maintain their regular lifestyle and social activities despite the seriousness of the situation. With regard to medical decision support, several participants said that this aspect was essentially under the control of physicians, while explaining that the OPN played a vital role in the comprehension of the "physicians' game (intervention) plan." Lastly, regarding the medium-term effects, it was reported that maintaining contact with the OPN reduced anxiety linked to separation and fear of recurrence. Several respondents described the availability of the OPN as a safety net.

UHC caregivers. Before implementation, the majority of caregivers believed that the OPN's role would foster care centred on cancer patient needs. Indeed, during implementation, they described an improved understanding of the needs of individuals with cancer and their families. They also thought that the OPN contributed to better coping with the illness and maintenance of a more stable psychological condition. According to them, the presence of the OPN also improved services organization. Nurses stressed improvements in teaching and continuity of care. Physicians reported that individuals with cancer were arriving better-prepared. Nurses and physicians also mentioned that the OPN helped to prevent complications. For the medium-term, caregivers believed that implementing the OPN's role would be beneficial for individuals with other types of cancer and that it could even be generalized to certain chronic illnesses. They suggested implementing the OPN's role in areas where the disease was complex, the volume of individuals with cancer high, multidisciplinary work possible and both clinicians and administrators were in favour of this approach. However, for some, the various stakeholders' roles must be further clarified in order to eliminate grey areas.

Network partners. For them, the OPN presence has generally been associated with positive changes. Several partners stated that the OPN had a positive impact on their feeling of competence in working with the head and neck population. They added that the information search was easier, that they felt supported in their interventions and this, in turn, made them feel as if they were providing continuing care in cooperation with UHC caregivers. Regarding the medium-term, following the example of UHC caregivers, several of the partners described that the main anticipated impact was an improvement in the quality and continuity of care, both from a clinical and relational perspective. These caregivers also believed that the OPN's presence could reduce hospital admissions, thus affecting services organization. As she is a member of a team specializing in oncology, the OPN can inform them, orient them and confirm the validity of their interventions. The OPN also fostered the development and maintenance of an efficient head and neck service hub.

\section{Discussion}

The main objective was to put forward a profile of the OPN's role and functions, and provide a qualitative description of the implementation process and its effects.

\section{Description of the OPN's role and functions}

Specifically, the first objective consisted of describing the OPN's role and functions as perceived by the stakeholders and the evolution of their perceptions over time. First, we noted that the representation of the role evolved from a theoretical perspective stemming from information sessions where a potential profile was presented, to a factual representation based on the stakeholders' field interactions with the OPN. This finding fits in rather well with what was anticipated. It would suggest that the various strategies put into place by the area committee during the preparatory phase fostered the development of a vision deriving from practical experiences. Paving the way played an important role that was welldescribed in the evaluation of the "navigation" program in Nova Scotia (Cook, 2005).

Among all of the OPN's functions, support is by far the most important for individuals with cancer and their families. It mostly involves providing information that is well-adapted to the care trajectory and the nature of their needs. For caregivers, however, care coordination prevails. What was initially seen as a transition 
mechanism from one step to another became first a coordination tool, then an integration tool for the care for people living with cancer. Caregivers' concerns progressively moved toward responding to the needs of persons with cancer and their families, which is the PQLC's aim. However, the assessment function was more limited. This may be because no formal tool such as the electronic health record has yet been developed to provide access to the follow-up provided by the OPN. This may also indicate that this function could be further developed. However, a few caregivers consulted clinical tools such as the initial assessment form, which is part of the health record and contained valuable content (Plaisance \& de Serres, 2004). To conclude, the principal stakeholders have mainly reported three of the four functions anticipated for the OPN, and the role is evolving toward the desired model (as taken from the PQLC).

\section{Description of the OPN's effect on the process of adaptation}

The second objective was to evaluate the effects on the process of adaptation to illness in individuals with cancer and their families. Results suggest that the OPN's presence is associated with positive changes in their attitude to the illness and with reinforcing coping strategies in individuals with cancer and their loved ones. Individuals with cancer explained that the OPN's presence helped them to better understand their health problem, treatments and care plan. At the same time, caregivers gained a better understanding of this population's needs. The synergy between the information given and that received seemed to represent the critical element of the process of adaptation to illness and treatments. This interpretation is consistent with the statements by Mongeau, Foucault, Ladouceur, and Garon (2002) and suggested that an important part of the support reported by patients and their families come from 1) the provision of information in a timely fashion and in response to the needs, and, 2) the assurance that information will be received in the future, should the need arise.

\section{Description of the OPN's effect \\ on the continuity of care and services}

The third objective aimed at evaluating the effects of implementing the OPN's role on continuity of care and services provided by the oncology interdisciplinary team at the UHC and the external partners of the health care network. According to the statements gathered, it would appear that the OPN's arrival has helped to improve the communication between caregivers and to facilitate interdisciplinary work. The presence of an OPN helped rally caregivers around an interdisciplinary care plan by centring the intervention objectives on the needs of the individuals with cancer and their families, not those of the caregivers. These observations are related to the phenomenon described when implementing nine OPNs in another region of Quebec (Plante, 2005) and underscore the importance of communication skills for OPNs.

Besides an improvement to interdisciplinary work, UHC caregivers and partners also perceived positive impacts at the level of the organization and continuity of care. However, caregivers deplored the fact that this improvement did not find an echo at the interinstitutional level nor in the community, especially with family physicians who made little contact with the OPN. The implementation process seems to have been restricted to the UHC. This situation may be due to the fact that, at the time of the study, implementation of the PQLC was just in its infancy. Despite this limitation, network partners described positive impacts for implementing an OPN on the level of communication between a specialized team and a front-line team.

\section{Limitations of the descriptive study}

With the exception of role duplication and skill area overlap, the majority of participants mentioned few disadvantages to implementing the OPN role. This result remains surprising. In the health care network with its work overload and limited resources, it is possible that some associated potential disadvantages, such as the possibility that the OPN had taken on overly problematic cases, may have been masked by the perceived advantages. It may also be that where caregivers are concerned, the stress on the positive side was associated with a desire to see this new role maintained after the evaluation process, a thought often expressed during the interviews. These factors may have contributed to the under-reporting of perceived negative or undesired effects. However, the effort to include all stakeholder groups contributed to increasing the ecological validity, which reflects the dynamics of what is taking place in the field. Moreover, the triangulation of stakeholders increased data validity and scientific validity of the evaluation approach because it allowed us to cross-reference and check consistency among the perceptions of the various stakeholder groups (Poupart et al., 1997).

\section{Implications}

The verification of the first objective shows how important it is to properly pave the way and take into account the organization's internal dynamics before implementing such an organizational change. It also highlights several practical aspects, especially the holding of information and consultation sessions with the main stakeholders and the need to create a common vision. It confirms the importance of this common vision that places the well-being of the person with cancer at the centre of the project, which took on a concrete form thanks to the utilization of the PQLC, the favoured coordination tool. One still needs to know and understand it well.

The verification of the second objective establishes that the OPN has a beneficial effect on the process of adaptation in individuals with cancer and their families. The study helped further reflect on support services to put into place to foster the process of adaptation of the individual with cancer and his or her family. The study draws attention to the importance of individualized information that is delivered in a timely fashion and remains consistent from one source to another. Moreover, it encouraged the examination of the OPN's role in early detection and prevention of adjustment problems to cancer. While emphasizing the emotional and instrumental support function associated with the OPN's role, this study questions the concurrent assessment function and the possibility of including a more systematic screening for emotional distress, suicide risk assessment and referral for mental health problems. In our opinion, the screening function and referral mechanisms remain to be specified.

Finally, the verification of the third objective yielded some leads to improve continuity and quality of care by highlighting the need to broaden the definition of continuity. The OPN's role and functions must not be limited to coordinating the care trajectory. Ensuring service continuity is a relevant function both at the stages of the diagnosis of the disease and its curative phase. During certain transitions, for example when moving from a curative to a palliative approach, the relational continuity takes all its meaning. Similarly, results demonstrate the importance of interdisciplinary work and integrated approaches. Without a team philosophy centred on the needs of individuals with cancer and their families, the OPN's usefulness can be questioned. While stakeholders favourably view the OPN's role as a partial solution to organizational constraints, they wonder how feasible it is to keep on multiplying the number of caregivers. It is obvious that the OPN cannot constitute a single solution to continuity problems. In that vein, the PQLC sets forth two other measures that are: access to electronic records and definition of services hubs within which the OPN's role can be anchored. 


\section{Conclusion}

This implementation process justifies the OPN's role and suggests several procedures to be taken into account when adding one such stakeholder to a multidisciplinary team. Results underscore: 1) the possibility of integrating the OPN's role within a team specializing in oncology, 2) the association between the presence of an OPN and facilitating the process of adaptation to illness, and 3) the

\section{References}

Agretelis, J.M. (1997). Commentary: The nurse's role in the management of the treatment plan. Oncology Nursing Forum, 24, 451-452.

Ambler, N., Rumsey, N., Harcourt, D., Khan, F., Cawthorn, S., \& Barker, J. (1999). Specialist nurse counsellor interventions at the time of diagnosis of breast cancer: Comparing "advocacy" with a conventional approach. Journal of Advanced Nursing, 29, 445453.

Anderson-Loftin, W. (1999). Nurse case managers in rural hospitals. Journal of Nursing Administration, 29, 42-49.

Bottomley, A., \& Jones, L. (1997). Breast cancer care: Women's experience. European Journal of Cancer Care, 6, 124-132.

Comité consultatif sur le cancer. (1997). Programme québécois de lutte contre le cancer: pour lutter efficacement contre le cancer, formons équipe. Québec, QC: Ministère de la Santé et des Services sociaux.

Conger, M., \& Craig, C. (1998). Advanced nurse practice: A model for collaboration. Nursing Case management, 3, 120-127.

Cook, S. (2005, Février). Cancer patient navigation in Nova Scotia. Présentation au colloque Navigation and self-management: Innovative approaches to managing chronic conditions, Richmond, BC.

Corner, J. (1999). Cancer nursing: A leading force for health care. Journal of Advanced Nursing, 29, 275-276.

de Serres, M., \& Beauchesne, N. (2000). L'intervenant pivot en oncologie: un rôle d'évaluation, d'information et de soutien pour le mieux-être des personnes atteintes de cancer. Rapport de recherche. Québec, QC: Conseil québécois de lutte contre le cancer, Ministère de la Santé et des Services sociaux.

de Serres, M., \& Rochette, M. (2004, Mars). Les infirmières pivots en oncologie au Québec: l'état de situation en 2004. Présentation au Forum sur l'infirmière pivot en oncologie, Longueuil, QC.

Farber, J., Deschamps, M., \& Cameron, R. (2002). Analyse et évaluation de la fonction d'intervenant pivot en réponse aux besoins qu'éprouvent les Canadiennes atteintes d'un cancer du sein sur les plans de l'information, de l'éducation et de l'aide à la prise de décision. Rapport de recherche. Québec, QC: Initiative sur le cancer du sein, Santé Canada.

Fillion, L., \& Morin, D. [En ligne - 2003]. Optimiser la qualité de l'exercice infirmier en soins palliatifs dans le contexte du virage ambulatoire. http://www.chrsf.ca/funding_opportunities/ ogc/fillion_f.php

Fillion, L., Morin, D., \& Saint-Laurent, L. (2000). Description du concept de l'intervenant pivot: approche théorique et qualitative. Rapport de recherche. Québec, QC: Université Laval.

Fitch, M., \& Mings, D. (2003). Les soins infirmiers en oncologie en Ontario: définition des rôles infirmiers. Canadian Oncology Nursing Journal, 13, 36-44.

Fraser, A. (1995). Pour une meilleure compréhension des besoins des personnes atteintes de cancer. Québec, QC: Comité consultatif sur le cancer, Ministère de la Santé et des Services sociaux.

Goldberg, N. (1994). The importance of timing in breast cancer treatment. Cancer Practice, 2, 450-451. improvement of interdisciplinary work and continuity of care. Several recommendations are formulated, among which is the importance of situating the implementation process from an organizational change perspective that recognizes the contribution of all stakeholders involved.

Groupe de travail sur les soins de soutien et la réadaptation au niveau du cancer. [En ligne - 2002]. Stratégie canadienne de lutte contre le cancer. Rapport de recherche. http://www.cancer.ca: Société canadienne du cancer.

Haddock, K.S., Johnson, P.K., Cavanaugh, J., \& Stewart, G.S. (1997). Oncology case-management linking structure and process with clinical and financial outcomes. Nursing Case Management, 2(2), 44-48.

Howell, D., \& Jackson, J. (1998). Making cancer bearable: The Interlink Community Cancer Nurses Model of Supportive Care. Canadian Oncology Nursing Journal, 8, 222-228.

Kugaya, A., Akechi, T., Okamura, H., Mikami, I., \& Uchitomi, Y. (1999). Correlates of depressed moods in ambulatory head and neck cancer patients. Psycho-Oncology, 8, 494-499.

Mongeau, S., Foucault, C., Ladouceur, R, \& Garon, R. (2002). La traversée du cancer du point de vue des conjoints endeuillés. Rapport de recherche. Verdun, QC: Fondation du Centre hospitalier Angrignon.

National Cancer Institute of Canada. [En ligne - 2005]. Canadian cancer statistics 2005. http://www.ncic.cancer.ca

Nickel, J.T., Salsberry, P.J., Caswell, R.J., Keller, M.D., Long, T., \& O'Connell, M. (1996). Quality of life in nurse case management of persons with AIDS receiving home care. Research in Nursing and Health, 19, 91-99.

Nolan, M.A., Harris, A., Kufta, A., Opfer, N., \& Turner, H. (1998). Preparing nurses for the acute care case manager role: Educational needs identified by existing case managers. Journal of Continuing in Nursing, 29, 130-134.

Patton, M.Q. (1997). Utilization-focused evaluation: the new century text (3rd ed.). London (UK): Sage publications.

Poupart, J., Deslauriers, J.P., Groulx, L.H., Laperrière, A., Mayer, R., et Pires. (1997). La recherche qualitative. Enjeux épistémologiques et méthodologiques, Montréal, Gaëtan Morin éditeur.

Plaisance, L., \& de Serres, M. (2004, Mars). L'évaluation initiale: moment clé pour des interventions efficaces, le plus près possible du diagnostic. Présentation au Forum sur l'infirmière pivot en oncologie, Longueuil, QC.

Plante, A. (2005, Février). Empowerment and team work - A complex symptoms management care model. Présentation au colloque Navigation and self-management: innovative approaches to managing chronic conditions, Richmond, BC.

Robert, G. (2000). Bien préparer les changements organisationnels. Revue québécoise de psychologie, 21, 37-51.

Strauss, A.L., \& Corbin, J.M. (1990). Basics in qualitative research: Grounded theory procedures and techniques. Newbury Park, CA: Sage Publications.

Turgeon, J., Dumont, S., St-Pierre, M., Sévigny, A., \& Vézina, L. (2004). La problématique de la continuité des soins en oncologie. Rapport de recherche soumis à la Fondation canadienne de recherche sur les services de santé. Québec, QC: Université Laval.

Weekes, D.P. (1997). Commentary: Socio-cultural considerations. Oncology Nursing Forum, 24, 452. 